\title{
Medical Management of Males and Females in Return to Learn and Return to Play after Concussion: An Observational Study
}

\author{
Amy Hassen ${ }^{1}$, Ariel Klingaman ${ }^{1}$ and Jennifer Reneker ${ }^{1,2 *}$ \\ ${ }^{1}$ Physical Therapy Program, Walsh University, USA \\ ${ }^{2}$ Department of Physical Therapy, University of Mississippi Medical Center, USA
}

*Corresponding author: Jennifer C Reneker, Department of Physical Therapy, School of Health Related Professions, University of Mississippi Medical Center, 2500 North State Street, Jackson, MS 39216, USA, Tel: 601-984-6326, Fax: 601815-1715, E-mail: JReneker@umc.edu

\begin{abstract}
This study aimed to 1) Describe differences in medical management between males and females after a concussion, and 2) Explore factors associated with the number of days to Return to Learn (RTL) and Return to Play (RTP). 111 subjects, aged 12-19 with sports-related concussion participated. Independent samples t-tests, chi-square tests, and Poisson regression were used to determine the predictors for the days to RTL and RTP. Females received recommendations for school and sports restrictions more often than males ( $p<0.03$ ), were held out of sports twice as long as males ( $p=0.002)$, and did not have full RTL 3 times longer than males $(p<0.001)$. Post-Concussion Symptom Score at baseline was the only significant predictor of the number of days to RTL $(p<0.001)$ and sex was the only significant predictor of the number of days to RTP $(p=0.04)$. The results of this study demonstrate specific medical management differences between the sexes after concussion. Understanding these differences may aid in the development of sex specific guidelines for RTL/RTP.
\end{abstract}

\section{Keywords}

Sports-related concussion, Adolescent athlete, Return to learn, Return to play, Academic accommodations, School restrictions

\section{Main Body}

Medical management of males and females in return to learn and return to play after concussion: An observational study.

\section{Introduction}

Although an estimated 1.1-1.9 million sports- and recreation-related concussions occur annually amongst United States youth ages 18 and under [1], limited evidence is available to guide medical management. This topic is of growing concern as there has been an overall $60 \%$ increase in the incidence of reported concussion between 2007 and 2014, with the largest increases occurring in the $10-14$ year-old (143\%) and $15-19$ year-old (87\%) age ranges [2]. Additional data examining emergency department visits for concussion between 2001 and 2005 amongst United States youth aged 8 to 19 found that approximately half of all visits for concussion were sports-related [3]. Since evidence suggests the youth population takes longer to recover from concussion than college athletes [4-7], clinical guidance is needed to address the aftercare of these types of injuries.

The management of concussion for adolescents as athletes has been described and is routinely recognized through the establishment of "Return to Play" (RTP) guidelines [8], however, limited attention has been given to the management of adolescents as students returning to the school environment, or "Return to Learn" (RTL) $[9,10]$. Specifically, research addressing the resumption of typical school participation in relation to the resumption of participation in sports remains lacking. Evidence has suggested that up to $43.5 \%$ of concussion cases return to play prematurely and $44.7 \%$ of concussion cases return to learn prematurely as demonstrated by a recurrence or worsening of symptoms with the return process [11]. Identifying the optimal time to 
return a student-athlete to the learning environment is a delicate balance. On one hand, there is a need to prevent the worsening of concussion symptoms by over stimulating a concussed brain $[12,13]$ and on the other, time out of school may increase the potential production of anxiety from missing school $[10,14]$.

Recently, a protocol for RTL was developed through a scoping literature review and synthesis of stakeholder opinions [15]. This protocol endeavored to mirror the basic steps of the five stage RTP guidelines, with progression from full rest to full days of school. At this time, the implementation of this protocol, evidence of its effectiveness, or an evaluation of the timeframe for RTL has not yet been published. Additionally, there are no clear guidelines regarding what cognitive rest entails for students and how long it should last [11]. Similar to RTP, the research does suggest that RTL needs to be individualized and progressed by symptom resolution $[15,16]$. The following signs and symptoms may be associated with a lengthier recovery: history of prior concussion; greater number of concussions, severity or duration of symptoms post-concussion; female sex; a history of a learning disorder; attention deficit disorders; or mood disorders $[9,17]$, and may impact the timeframe for RTL.

As guidelines develop to aid health care practitioners, school administrators, and parents in making decisions related to the learning environment, a better understanding of how the medical management of RTL relates to the established protocol for RTP is needed, particularly in examining differences in RTL between male and female athletes following a concussion. Any differences in recovery patterns between the sexes would provide valuable information regarding the approach for RTL and RTP; thus enabling the development of evidence based clinical guidelines, tailored to any unique features between males and females. The purpose of this study was to 1) Describe differences between males and females in medical management and timeline for RTL and RTP and 2) Explore the demographic, premorbid, and clinical factors associated with the number of days to RTL and RTP.

\section{Methods}

\section{Study design}

This is a secondary analysis of data collected as a part of a prospective observational study utilizing participants seen as patients at Akron Children's Hospital $(\mathrm{ACH})$ Sports Medicine Center in Akron, Ohio. Two previous publications have utilized data from this cohort of participants $[18,19]$. The Institutional Review Board at Akron Children's Hospital and the Human Subjects Review Board at Walsh University approved the original research. Informed consent was granted by the parent or legal guardian of each participant or was obtained by the participant if he/she was 18 or older. Assent was obtained from those participants under the age of 18 .

\section{Setting}

Participants were invited to enroll in this study if they presented to the ACH Sports Medicine Center between August 2013 and April 2014. Participants were followed from the first medical visit for management after a sports-related concussion through discharge from medical care. Data was collected through a structured patient interview conducted at the sports medicine center and from medical records related to the sports medicine visits. The data collected included documented concussion management strategies and prescribed activity restrictions.

\section{Participants}

A convenience sample was utilized for this study, which consisted of adolescent athletes ages 12-19 with a diagnosis of sports-related concussion. Participants were considered for inclusion in the sample if they sustained a concussion while participating in a sports activity. Participants were not considered for inclusion if concussion was sustained through a motor vehicle accident or other etiology unrelated to sports activity (recreational or organized). Participants included adolescents with a history of concussion, as well as those with no history of concussion.

\section{Variables and data measurement}

The data collected for each participant included: date of birth, sex, date of current concussion, date of initial assessment at the sports medicine center, and sport in which concussion occurred. Each participant and/or parent reported on the intake history and physical form, as per standard clinic practice, in answer to this question: "Have you ever had medical treatment for any of the following: headaches/migraines, and psychiatric condition (depression, anxiety)?". Participants were also asked to indicate if they had ever had a previous concussion or Attention Deficit Hyperactivity Disor$\operatorname{der}(A D H D / A D D)$. The total Post-Concussion Symptom Scale (PCSS) was recorded at the initial visit.

The PCSS is a 22-item self-report symptom scale routinely utilized to track concussion severity and resolution of symptoms throughout recovery. The participant, on a 7-point Likert scale, ranks each of the 22 items from 0 (not a symptom the participant is experiencing) to 6 (severe symptom the participant is experiencing). The symptoms presented on the PCSS represent constructs including physical complaints (e.g. headache, dizziness, vision problems); emotional symptoms (e.g. sadness, irritability); sleep disturbances (e.g. sleeping more than usual, trouble falling asleep); and cognitive complaints (e.g. mentally foggy, difficulty remembering) [20,21].

Data was also collected regarding any prescribed recommendations for physical and mental rest, restrictions from school, accommodations within school, and restrictions from physical activity. Physician recommen- 
dations of "no school" or "half day in school", were categorized as school restrictions. If any recommendations were made regarding an alteration in student participation while at school, the participant was regarded as requiring academic accommodations. Examples of academic accommodations included any of the following: receiving extra time to complete assignments, extra time for taking tests, reduced academic workload, or modified assignments. Physician recommendations of "no sports or physical education class", or "able to begin low intensity physical activity (only)", were categorized as sports restrictions. Each of these variables was collected at the initial medical appointment and at each follow-up appointment through discharge.

The operational definition for RTL was developed to mirror the well-established final stage for RTP [8]. For the purposes of this study, RTL was defined as full ac- ademic participation with no restriction of school attendance and no academic accommodations. RTP was defined as full sports participation with no restrictions (Stage 5 of a RTP progression according to the Zurich guidelines) [8]. The number of days of treatment prior to RTP and RTL were defined as the time from the initial medical appointment to either return to play or return to learn.

\section{Statistical analyses}

Statistical analyses were completed with Statistical Analysis Software (SAS) 9.4. Descriptive statistics for the sample were calculated. Independent samples t-tests and chi-square tests were utilized to determine if there were differences between males and females in participant characteristics, past medical history, PCSS scores at the initial sports medicine visit, variables measuring medical recommendations provided for academic and

Table 1: Descriptive statistics for the sample.

\begin{tabular}{|c|c|c|c|c|c|}
\hline & $\begin{array}{l}\text { Males } \\
(n=76)\end{array}$ & $\begin{array}{l}\text { Females } \\
(n=35)\end{array}$ & p-value & t-value & $\begin{array}{l}\text { Effect size } \\
\text { (d or OR) }\end{array}$ \\
\hline \multicolumn{6}{|l|}{ Participant characteristics } \\
\hline Age & $15.0(1.5)$ & $15.1(1.7)$ & 0.73 & -0.34 & 0.07 \\
\hline Sport & & & $<0.01$ & & NC \\
\hline Football & $48(64.9 \%)$ & $1(2.9 \%)$ & & & \\
\hline Soccer & $6(8.1 \%)$ & $9(25.7 \%)$ & & & \\
\hline Basketball & $7(9.5 \%)$ & $10(28.6 \%)$ & & & \\
\hline Wrestling & $3(4.1 \%)$ & $1(2.9 \%)$ & & & \\
\hline Swimming & $1(1.4 \%)$ & $1(2.9 \%)$ & & & \\
\hline Rugby & $1(1.4 \%)$ & 0 & & & \\
\hline Hockey & $1(1.4 \%)$ & 0 & & & \\
\hline Cheerleading & 0 & $3(8.6 \%)$ & & & \\
\hline Volleyball & 0 & $2(5.7 \%)$ & & & \\
\hline Baseball/Softball & 0 & $1(2.9 \%)$ & & & \\
\hline Lacrosse & 0 & $1(2.9 \%)$ & & & \\
\hline Other & $7(9.5 \%)$ & $6(17.1 \%)$ & & & \\
\hline \multicolumn{6}{|l|}{ Participant medical history } \\
\hline History of previous concussion (yes) & $22(29.0 \%)$ & $14(40 \%)$ & 0.25 & & 1.64 \\
\hline History of headaches (yes) & $15(19.7 \%)$ & $12(34.3 \%)$ & 0.10 & & 2.12 \\
\hline Psychiatric condition (yes) & $1(1.3 \%)$ & $7(20.0 \%)$ & $<0.01$ & & 18.75 \\
\hline History of ADHD (yes) & $10(13.2 \%)$ & $3(8.6 \%)$ & 0.49 & & 0.62 \\
\hline \multicolumn{6}{|l|}{ Clinical characteristics } \\
\hline Number of days between concussion and assessment & $8.6(6.3)$ & $9.8(15.1)$ & 0.65 & -0.46 & 0.09 \\
\hline PCSS score at initial visit & $15.4(16.1)$ & $34.7(25.4)$ & $<0.01$ & -3.06 & 0.63 \\
\hline \multicolumn{6}{|l|}{ Medical recommendations provided } \\
\hline Academic accommodations (yes) & $44(57.9 \%)$ & $31(88.6 \%)$ & 0.01 & & 5.28 \\
\hline School restrictions (yes) & $42(55.3 \%)$ & $29(82.9 \%)$ & 0.03 & & 3.68 \\
\hline No school/work & $23(30.7 \%)$ & $22(66.7 \%)$ & & & \\
\hline Shortened day & $18(24.0 \%)$ & $6(18.2 \%)$ & & & \\
\hline Sports restrictions (yes) & $61(80.3 \%)$ & $35(100 \%)$ & $<0.001$ & & 17.89 \\
\hline No sports/physical education & $52(68.4 \%)$ & $32(91.4 \%)$ & & & \\
\hline Low intensity physical activity only & $9(11.8 \%)$ & $2(5.7 \%)$ & & & \\
\hline \multicolumn{6}{|l|}{ Number of days of treatment until: } \\
\hline Return to play & 18.0 & 44.3 & 0.002 & -3.35 & 0.68 \\
\hline Return to learn & 8.0 & 23.9 & $<0.001$ & -4.48 & 0.92 \\
\hline
\end{tabular}

Attention Deficit Hyperactivity Disorder = ADHD; Post Concussion Scale = PCSS; Psychiatric condition included anxiety disorder; depression; and bipolar disorder; History of headaches includes history of migraines; T-tests completed on continuous variables, Chi-Square analyses completed on categorical/binomial variables. Significant differences are based on alpha < 0.05 ; point estimate of effect sizes for differences between males and females calculated as $d$ for continuous variables based on the t-statistic and Odds Ratios for dichotomous variables with males as the referent. 
sports restrictions, and the number of days of treatment prior to RTL and RTP. Additionally, effect sizes were calculated, including Cohen's d for continuous variables and odds ratios for dichotomous variables to express the size of the difference between males and females. Exploratory univariate Poisson regression was completed to determine relevant participant, past medical history, and clinical predictors on the number of days to RTL and RTP. Univariate associations with a p-value of 0.10 or less were added to each final multivariable Poisson model for RTL and separately for RTP. Multivariable Poisson regression was completed for RTL and RTP with all relevant predictors. Significant associations in the multivariable model were based on alpha $<0.05$.

\section{Results}

In total, 111 participants were enrolled into the study, 76 males and 35 females. The descriptive statistics for the sample can be found in Table 1. Most of the concussions occurred during participation in football for males and in basketball or soccer for females. A total of 36 participants had a history of a previous concussion, 27 had a history of headaches or migraines, and 13 total had a diagnosis of ADHD. According to chi-square analysis, the prevalence of these premorbid conditions was not significantly different between males and females ( $p$-values between 0.10 and 0.49). Eight of the participants had a history of a psychiatric condition with significantly more females reporting this than males $(p<0.01$; $\mathrm{OR}=18.75$ ). Participants reported to sports medicine for medical management at a mean of nearly 9 days after sustaining the concussion. At the initial medical visit, females demonstrated a significantly higher Post-Concussion Symptom Score than males $(p<0.01 ; d=0.63)$.

As a component of the medical management of concussion, females received recommendations for academic accommodations ( $p<0.01 ; O R=5.28)$, school restrictions (no school or a shortened day; $p=0.03$; OR $=3.68$ ) and sports restrictions (including no sports/physical education or allow only low intensity physical activity) significantly more often than males ( $p=<0.001$; OR $=17.89$ ). In addition, on average, females were held out of full participation in sports more than twice as long as males $(p=0.002 ; d=0.68)$ and did not have full return to learn for a period almost 3 times longer than males ( $p$ $=<0.001 ; \mathrm{d}=0.92$ ) (Table 1).

Considering the predictors of the number of days to RTL, after univariate analysis, sex, history of headaches, psychiatric condition, and PCSS score at the initial medical visit were included in the final regression model (Table 2). PCSS score at the initial medical visit was the only significant predictor of the number of days to RTL $(p<$ 0.001 ). Considering the predictors of the number of days to RTP, after univariate analysis, sex, history of previous concussion, psychiatric condition, and PCSS score at the initial medical visit were included in the final regression model. Here, sex was the only significant predictor of the number of days to RTP $(p=0.04)$.

\section{Discussion}

Our results indicate that females were provided academic accommodations, school restrictions, and sports restrictions more frequently than their male counter parts prior to returning to full scholastic or athletic participation. In addition, females were held out of sports and school participation significantly longer than the males in this study. Similar to the findings of this current study, existing literature supports the notion of females faring worse when related to concussions. Females have a higher rate of concussions in high school sports played by both sexes $[22,23]$. Additionally, female high school and college athletes have more post-concussion symptoms and are 1.7 times more likely to be cognitively impaired after a concussion [24]. Overall, a recent meta-analysis reveals that traumatic brain outcomes are worse in females than males [25].

The differences in symptomatic presentation and timeline for recovery in males and females is neither fully understood nor consistently reported in the literature. One study on 10 to 18 -year-olds found that although females experienced greater symptoms pre-concussion and in the acute post-concussive state, they experienced similar recovery times as compared to males [26]. In contrast, in a study on a sample of 18-23 yearolds, no significant difference was found between males and females in symptom presentation initially; however, male subjects were more likely than female subjects to be symptom free at 4 weeks [27]. In this present study,

Table 2: Predictors of number of days to Return to Learn (RTL) and Return to Play (RTP).

\begin{tabular}{|l|l|l|l|}
\hline & Parameter estimate & SD (95\% CI) & p-value \\
\hline Number of days to Return-To-Learn & & & 0.06 \\
\hline Sex (female) & 0.654 & $0.277(0.110,1.200)$ & 0.99 \\
\hline History of headaches (yes) & 0.001 & $0.218(-0.426,0.429)$ & 0.93 \\
\hline Psychiatric condition (yes) & -0.029 & $0.315(-0.646,0.588)$ & $<0.001$ \\
\hline PCSS score at initial visit & 0.016 & $0.005(0.007,0.026)$ & 0.04 \\
\hline Number of days to Return-To-Sport & & & 0.12 \\
\hline Sex (female) & 0.894 & $0.297(0.312,1.476)$ & 0.74 \\
\hline Previous history of concussion (yes) & 0.427 & $0.2528(-0.021,0.874)$ & 0.77 \\
\hline Psychiatric condition (yes) & 0.085 & $0.252(-0.409,0.578)$ & $0.006(-0.013,0.010)$ \\
\hline PCSS score at initial visit & -0.002 & & 0.074 \\
\hline
\end{tabular}

Post Concussion Scale = PCSS; Multivariable Poisson regression models presented. Significant values based on alpha $<0.05$. 
PCSS score at the initial medical visit was the only significant predictor of the number of days to RTL, with sex held constant. This potentially indicates that within our sample, RTL decisions are based on clinical factors separate from the patient's sex. In contrast, sex was the only significant predictor of the number of days RTP, with females taking significantly longer to return to play than males, with PCSS score held constant.

There are multiple potential reasons for the differences in outcome related to RTP between male and female athletes as described in this present study. From an anatomical and physiological perspective, the sexes have different intrinsic abilities to withstand blows to the head-neck complex. Research findings have demonstrated that females have greater head-neck angular acceleration and displacement than males even though they initiate muscle activity earlier and use a greater proportion of their total available muscle activity. These differences may be explained by less isometric strength, head mass and neck girth than their male counterparts [28-31]. On average, this difference in anatomy may result in more severe functional brain injuries in females than males. Thus, females would require a longer time to attain a level of overall recovery necessary for a medical professional to release the athlete for RTP.

Hormonal differences may also contribute to the differences seen with recovery. Beginning at baseline, females are $43 \%$ more likely than males to report symptoms [32]. Females may never become asymptomatic due to the role of the menstrual cycle as many of the symptoms females experience at baseline (headache, difficulty concentrating, emotional symptoms, and energy/sleep disturbances) are associated with the menstrual cycle $[33,34]$. Finally, there may be cultural influences that lead to greater reporting of concussion symptoms in female versus male athletes, with males avoiding or under-reporting symptoms $[21,35,36]$. Conversely, female athletes may have more concern for their future health and may be more forthcoming in their reporting [25]. Thusly, hormonal influence and behavioral reporting in females may intensify the expressed severity of the concussion. Because recovery is dependent upon patient report, these differences by sex could certainly influence when a medical provider is comfortable granting RTP and may explain, in part, reasons why females are held out from RTP longer than males. Further research on anatomical, hormonal and cultural influences on the difference in concussion recovery time between the sexes is warranted to explore and better understand these potential relationships. This knowledge may aid in the creation of sex specific guidelines for RTL and RTP. In the meantime, providers may need to look beyond symptoms to the potential cause of the symptoms (anatomical, hormonal, cultural), particularly in female patients, to aid in determination of appropriate RTL and RTP timelines.

\section{Limitations}

Although the data for the original research study were collected prospectively, the study was conducted at a medical clinic where a cohort of physicians and nurse practitioners made medical management decisions pragmatically and entered these decisions into a medical record. Although collected from one sports medicine center, there was no standardization of the decision-making processes between the providers, which creates the potential for differing decision-making drivers related to return to sport/school. Additionally, there were likely differences in documentation practices since the purpose of the documentation was medical, not research-oriented. Therefore, the results may not be easily generalizable to another medical clinic or cohort. Additionally, the outcomes associated with this sample are likely not representative of all student athletes who sustain a sports-related concussion because participants in this study were enrolled based on seeking medical management at a sports medicine center for their concussion symptoms. There may be important differences between parents and athletes who seek medical management and those who do not. Lastly, it should be noted that because there is a clear difference in the types of sports represented by males and females within this sample, it is possible that some of the differences that were found in this study are related to sport and not necessarily only sex. This was unable to be further explored.

\section{Conclusion}

As sports-related concussion remains a prevalent concern for adolescent athletes, standards for RTL must continue to be developed and monitored in a similar way to the guidelines and regulations in place for RTP. Our findings uncover differences in recovery trajectory from a concussion between males and females along with the factors influencing the timeframes for return to participation in school and sports. This research may help to inform the development of additional evidence-based guidelines for adolescents and children with sports-related concussion.

\section{Conflicts of Interest and Source of Funding}

This research did not receive any specific grant from funding agency in the public, commercial, or not-for-profit sectors. There are no potential conflicts to disclose.

\section{Acknowledgements}

The authors would like to thank Akron Children's Hospital and Joseph Congeni, MD for support and resources to carry out this research.

\section{References}

1. Bryan MA, Rowhani-Rahbar A, Comstock RD, Rivara F, Seattle Sports Concussion Research Collaborative (2016) Sports- and Recreation-Related Concussions in US Youth. Pediatrics 138: e20154635. 
2. Zhang AL, Sing DC, Rugg CM, Feeley BT, Senter C (2016) The Rise of Concussions in the Adolescent Population. Orthop J Sports Med 4.

3. Bakhos LL, Lockhart GR, Myers R, Linakis JG (2010) Emergency department visits for concussion in young child athletes. Pediatrics 126: e550-e556.

4. Sim A, Terryberry-Spohr L, Wilson KR (2008) Prolonged recovery of memory functioning after mild traumatic brain injury in adolescent athletes. J Neurosurg 108: 511-516.

5. Moser RS, Schatz P, Jordan BD (2005) Prolonged effects of concussion in high school athletes. Neurosurgery 57: 300-306.

6. Field M, Collins MW, Lovell MR, Maroon J (2003) Does age play a role in recovery from sports-related concussion? A comparison of high school and collegiate athletes. J Pediatr 142: 546-553.

7. Fazio VC, Lovell MR, Pardini JE, Collins MW (2007) The relation between post concussion symptoms and neurocognitive performance in concussed athletes. NeuroRehabilitation 22: 207-216.

8. McCrory P, Meeuwisse W, Aubry M, Cantu B, Dvorak J, et al. (2013) Consensus statement on concussion in sport--the 4th International Conference on Concussion in Sport held in Zurich, November 2012. Clin J Sport Med 23: 89-117.

9. Harmon KG, Drezner JA, Gammons M, Guskiewicz KM, Halstead M, et al. (2013) American Medical Society for Sports Medicine position statement: concussion in sport. $\mathrm{Br}$ J Sports Med 47: 15-26.

10. DeMatteo C, Stazyk K, Giglia L, Mahoney W, Singh SK, et al. (2015) A Balanced Protocol for Return to School for Children and Youth Following Concussive Injury. Clin Pediatr (Phila) 54: 783-792.

11. Carson JD, Lawrence DW, Kraft SA, Garel A, Snow CL, et al. (2014) Premature return to play and return to learn after a sport-related concussion: physician's chart review. Can Fam Physician 60: 310-315.

12. Sady MD, Vaughan CG, Gioia GA (2011) School and the concussed youth: recommendations for concussion education and management. Phys Med Rehabil Clin N Am 22: 701-719.

13. Howell D, Osternig L, Van Donkelaar P, Mayr U, Chou LS (2013) Effects of concussion on attention and executive function in adolescents. Med Sci Sports Exerc 45: 1030-1037.

14. Purcell L, Harvey J, Seabrook JA (2016) Patterns of Recovery Following Sport-Related Concussion in Children and Adolescents. Clin Paediatr 55: 452-458.

15. DeMatteo C, McCauley D, Stazyk K, Harper J, Adamich J, et al. (2015) Post-concussion return to play and return to school guidelines for children and youth: a scoping methodology. Disabil Rehabil 37: 1107-1112.

16. Santiago S (2016) Adolescent Concussion and Return-toLearn. Pediatr Ann 45: e73-e75.

17. Makdissi M, Darby D, Maruff $P$, Ugoni A, Brukner $P$, et al. (2010) Natural history of concussion in sport: markers of severity and implications for management. Am J Sports Med 38: 464-471.

18. Moor HM, Eisenhauer RC, Killian KD, Henriques AA, Congeni JA, et al. (2015) The relationship between adherence behaviors and recovery time in adolescents after a sports-related concussion: an observational study. Int J Sports Phys Ther 10: 225-233.

19. Reneker J, Cheruvu V, Yang J, Cook CE, James MA, et al. (2015) Differential diagnosis of dizziness after a sports-re- lated concussion based on descriptors and triggers: an observational study. Inj Epidemiol 2: 22.

20. Kontos AP, Elbin RJ, Schatz P, Covassin T, Henry L, et al. (2012) A revised factor structure for the post-concussion symptom scale: baseline and postconcussion factors. Am J Sports Med 40: 2375-2384.

21. Lovell MR, Iverson GL, Collins MW, Podell K, Johnston $\mathrm{KM}$, et al. (2006) Measurement of symptoms following sports-related concussion: reliability and normative data for the post-concussion scale. Appl Neuropsychol 13: 166-174.

22. Gessel LM, Fields SK, Collins CL, Dick RW, Comstock RD (2007) Concussions among United States high school and collegiate athletes. J Athl Train 42: 495-503.

23. Dick RW (2009) Is there a gender difference in concussion incidence and outcomes? Br J Sports Med 43: 46-50.

24. Broshek DK, Kaushik T, Freeman JR, Erlanger D, Webbe F, et al. (2005) Sex differences in outcome following sports-related concussion. J Neurosurg 102: 856-863.

25. Farace E, Alves WM (2000) Do women fare worse? A metaanalysis of gender differences in outcome after traumatic brain injury. Neurosurg Focus 8: 6.

26. Ono KE, Burns TG, Bearden DJ, McManus SM, King H, et al. (2016) Sex-Based Differences as a Predictor of Recovery Trajectories in Young Athletes After a Sports-Related Concussion. Am J Sports Med 44: 748-752.

27. Henry LC, Elbin RJ, Collins MW, Marchetti G, Kontos AP (2016) Examining Recovery Trajectories After Sport-Related Concussion With a Multimodal Clinical Assessment Approach. Neurosurgery 78: 232-241.

28. Barnes BC, Cooper L, Kirkendall DT, McDermott TP, Jordan BD, et al. (1998) Concussion history in elite male and female soccer players. Am J Sports Med 26: 433-438.

29. Queen RM, Weinhold PS, Kirkendall DT, Yu B (2003) Theoretical study of the effect of ball properties on impact force in soccer heading. Med Sci Sports Exerc 35: 2069-2076.

30. Mansell J, Tierney RT, Sitler MR, Swanik KA, Stearne D (2005) Resistance training and head-neck segment dynamic stabilization in male and female collegiate soccer players. J Athl Train 40: 310-319.

31. Tierney RT, Sitler MR, Swanik CB, Swanik KA, Higgins $M$, et al. (2005) Gender differences in head-neck segment dynamic stabilization during head acceleration. Med Sci Sports Exerc 37: 272-279.

32. Brown DA, Elsass JA, Miller AJ, Reed LE, Reneker JC (2015) Differences in Symptom Reporting Between Males and Females at Baseline and After a Sports-Related Concussion: A Systematic Review and Meta-Analysis. Sports Med 45: 1027-1040.

33. Schmelzer K, Ditzen B, Weise C, Andersson G, Hiller W, et al. (2015) Clinical Profiles of Premenstrual Experiences Among Women Having Premenstrual Syndrome (PMS): Affective Changes Predominate and Relate to Social and Occupational Functioning. Health Care Women Int 36: 1104-1123.

34. Freeman EW, Halberstadt SM, Rickels K, Legler JM, Lin H, et al. (2011) Core symptoms that discriminate premenstrual syndrome. J Womens Health (Larchmt) 20: 29-35.

35. Colvin AC, Mullen J, Lovell MR, West RV, Collins MW, et al. (2009) The role of concussion history and gender in recovery from soccer-related concussion. Am J Sports Med 37: 1699-1704.

36. Covassin T, Elbin RJ (2011) The female athlete: the role of gender in the assessment and management of sport-related concussion. Clin Sports Med 30: 125-131. 